Revista de Investigación Educativa 24

enero-junio, 2017 | ISSN 1870-5308 | Xalapa, Veracruz

Instituto de Investigaciones en Educación | Universidad Veracruzana

Desigualdad educativa y su relación con la distribución del ingreso en los estados mexicanos

Educational inequality and its relationship to income distribution in the Mexican states

\author{
Dr. Antonio Favila Tello \\ Profesor-Investigador \\ Instituto de Investigaciones Económicas y Empresariales \\ Universidad Michoacana de San Nicolás de Hidalgo, México \\ antonio_favila@hotmail.com \\ Dr. José César Lenin Navarro Chávez \\ Profesor-Investigador \\ Instituto de Investigaciones Económicas y Empresariales \\ Universidad Michoacana de San Nicolás de Hidalgo, México \\ cesar126@hotmail.com
}

El presente trabajo aborda la temática de la desigualdad educativa en México cuantificando los coeficientes de Gini para la educación por entidad federativa para; posteriormente, estos datos se utilizan como insumo para indagar acerca de la relación entre la desigualdad en la distribución de los ingresos trimestrales por hogar y el grado de desigualdad educativa en estas entidades, utilizando para ello en método de mínimos cuadrados ordinarios. Los resultados sugieren la existencia de profundas brechas de desigualdad entre entidades, encontrándose las condiciones más adversas en Chiapas, Oaxaca, Guerrero y Michoacán, y una influencia menor de la desigualdad educativa sobre la desigualdad del ingreso en los estados mexicanos.

Palabras clave: Desigualdad educativa; Coeficiente de Gini; escolaridad; ingresos económicos. 
This paper addresses the issue of educational inequality in Mexico quantifying the Gini coefficients for education by state for 2015; then this data is used as input to inquire about the relationship between inequality in the distribution of quarterly household income and the degree of educational inequality in these entities , using the ordinary least squares method. The results suggest the existence of deep inequality gaps between entities, locating the most adverse conditions in Chiapas, Oaxaca, Guerrero and Michoacán. Also a minor influence of educational inequality on income inequality in the Mexican states is proven.

Keywords: Educational inequality; Gini coefficient; schooling; economic income.

\title{
Desigualdad educativa y su relación con la distribución del ingreso en los estados mexicanos
}

\author{
Educational inequality and its relationship to \\ income distribution in the Mexican states
}

\section{Introducción}

$\mathrm{U}_{\mathrm{e}}^{\mathrm{n}}$

no de los aspectos más ampliamente estudiados sobre la educación en México es el tocante a su distribución desigual. Si bien se han registrado avances significativos en la cobertura educativa en México y han surgido nuevos instrumentos (tales como las modalidades abiertas y a distancia de bachillerato y educación superior) para acortar las brechas de desigualdad educativa en la sociedad mexicana, el tema sigue siendo un objeto frecuente de estudio entre los académicos de distintas disciplinas.

Diversos trabajos han indagado alrededor de la desigualdad educativa en México, abordándola desde diferentes enfoques, particularmente relacionados con sus efectos sociales y con el daño que la misma representa al ejercicio pleno de los derechos de los colectivos que han resultado excluidos. 
Dentro de esta gama de temáticas, las relacionadas con sus efectos económicos han sido de las abordadas más ampliamente. El presente trabajo busca unirse a estas investigaciones aportando evidencias empíricas sobre la relación entre la desigualdad educativa, medida a través de la distribución de la escolaridad en las entidades federativas de México, y la desigualdad en la distribución del ingreso en las mismas.

En primer lugar, el trabajo pretende ser una actualización de otros que han medido la desigualdad educativa estatal, a través de la utilización del Coeficiente de Gini, y colaborar a que existan indicadores actualizados sobre la materia. En segundo lugar, este trabajo pretende probar la hipótesis que indica que una mayor desigualdad educativa es una característica estatal que contribuye a una distribución más desigual de los ingresos de los hogares.

Se comienza con una revisión de la literatura sobre el tema para posteriormente describir los instrumentos de medición utilizados. Después se comentan los resultados encontrados y se agregan algunas conclusiones.

\section{Revisión de literatura}

El estudio de la educación desde su perspectiva económica es relativamente reciente. La economía de la educación nació como una especialidad a mediados de los años cincuenta del siglo xx; sin embargo, la idea de la importancia de la educación en el incremento de la productividad y como determinante del crecimiento de la economía estuvo presente en el pensamiento económico desde tiempos de Adam Smith, aun cuando en aquel momento no se desarrollaron análisis detallados que lo corroboraran (Eicher, 1988).

Uno de los avances determinantes en el estudio de los aspectos económicos de la educación fue el desarrollo de la Teoría del Capital Humano. Su idea central consiste en considerar que el conjunto de habilidades que poseen los trabajadores son, en cierta forma, comerciables, es decir conforman una forma de capital que puede acumularse y aplicarse en la producción tal como ocurre con el capital físico. En consecuencia, el capital humano incluye aquellas características de los trabajadores, innatas o adquiridas, que contribuyen a incrementar su productividad (Acemoglu \& Autor, s.f).

A partir del desarrollo de dicha teoría, surgió un creciente interés en la cuantificación del capital humano y la medición de sus efectos. Con el tiempo, comenzó a 
ser ampliamente reconocido que el capital humano es un elemento fundamental para que la sociedad logre el progreso, tanto económico como social. Al incrementar la productividad de la población, de acuerdo con lo postulado en la Teoría del Capital Humano, no sólo se generan beneficios en la competitividad y en la capacidad de generación de riqueza de toda la economía, sino que también se coadyuva para la mayor retribución de los trabajadores a través de los salarios (Ordaz, 2007).

De tal forma, el Capital Humano cobró importancia no sólo desde la perspectiva económica, sino también desde la perspectiva social, al ser identificado como un agente relevante en el combate a la pobreza. Así, su estudio transitó de explicar la relación entre la formación y el crecimiento económico a esquemas de análisis con un carácter mucho más sociológico y multidisciplinar (Eicher, 1988).

Como producto de esta perspectiva más tendiente hacia lo social, se ha abordado con frecuencia el tema de la distribución desigual de la educación. Al respecto existe un consenso prácticamente generalizado sobre cómo el acceso igualitario a la educación es no solamente uno de los derechos humanos fundamentales, sino también un componente central del bienestar (Instituto Nacional para la Evaluación de la Educación [INEE], 2014; Thomas, Wang \& Fan, 2001).

La educación es considerada también como un medio para el aprovechamiento de las capacidades de la población, ya que si las capacidades y habilidades innatas de los individuos se distribuyen de manera normal, el hecho de que ciertos individuos o colectivos permanezcan marginados de las posibilidades de desarrollarse representa una pérdida para la sociedad en su conjunto (Thomas, Wang \& Fan, 2001).

Adicionalmente, diversos trabajos han demostrado que una distribución más igualitaria de la educación fomenta sociedades más saludables, incrementa la cohesión social y procura la participación ciudadana en los asuntos públicos (Meschi \& Scervini, 2010). Asimismo, el estudio de la desigualdad educativa constituye un mecanismo de evaluación y rendición de cuentas, ya que se presume que los avances o retrocesos que puedan observarse en la distribución de las credenciales educativas reflejan la eficiencia del sistema en la consecución de sus objetivos, así como en la gestión de sus recursos (Bracho, 1995).

Por lo anterior, el alcanzar una mayor igualdad educativa es una meta deseable y legítima de la sociedad, y el seguimiento periódico a los avances en la materia constituye un insumo importante para el mejor entendimiento de la problemática y para el diseño de políticas para su atención. 


\subsection{Definiendo a la desigualdad educativa}

Si bien no existe una definición unívoca de lo que es la desigualdad educativa, diversos autores han formulado posicionamientos alrededor de este concepto para aproximarse a su estudio, lo que permite identificar ciertas directrices generales adoptadas para su análisis. Por ejemplo, para Rodríguez (2009) "una educación equitativa es aquella que le permite a las personas tener acceso a una educación de similar cantidad y calidad, con independencia de su origen socioeconómico” (p. 1).

En opinión de Subirats, Gomá y Brugué (2005), la desigualdad educativa consiste en excluir a determinados individuos o colectivos del acceso, permanencia y aprovechamiento del sistema educativo.

Para Muñoz (2003), la desigualdad educativa sucede cuando una sociedad falla en la consecución de los siguientes objetivos: igualar las oportunidades de ingreso al sistema educativo para todos los individuos que posean las mismas habilidades; igualar estas mismas oportunidades entre individuos de distintos estratos socioeconómicos; igualar los resultados obtenidos por los estudiantes que dediquen a su educación la misma cantidad de tiempo y esfuerzo sin que en ello influya su origen social, y fomentar la adquisición de habilidades para el aprendizaje entre la población, utilizando estrategias diferenciadas que busquen igualar el desempeño de los beneficiarios.

Para Latapí (1983), la desigualdad en su sentido sociopolítico se refiere a la distribución diferenciada de los bienes sociales, entre los cuales es posible mencionar a la educación. La educación no solamente es un bien social que posee valor en sí mismo, sino que también lo tiene por funcionar como un medio para obtener otros bienes, no sólo de carácter material sino también de carácter inmaterial, tales como el prestigio o el poder.

Latapí (1983) propone tres planos desde los cuales es posible abordar el análisis de la desigualdad en la educación:

El primero es el plano socio-histórico, en el cual se explican las desigualdades en su relación con los proyectos políticos y los cambios que estos producen en las relaciones de poder dentro de la estructura social.

En el segundo plano, el análisis se centra en la política seguida por el Estado; en este plano, la capacidad diferenciada de presión de los diferentes grupos sociales genera que el gobierno adopte políticas concretas, principalmente a través de la asignación de recursos. En consecuencia, los procesos de igualación educativa se encuentran estrechamente ligados a las prioridades políticas que prevalezcan en ese momento. 
En el tercer plano, la desigualdad educativa se aborda desde la observación de indicadores concretos que reflejan el resultado del juego de fuerzas, las alianzas y los conflictos de los dos planos anteriores. Estos indicadores revelan las probabilidades de la población para obtener igualdad en el acceso y permanencia en la educación formal, igualdad de resultados académicos e igualdad de resultados externos al sistema educativo (tales como la ocupación y el ingreso).

\subsection{Retrospectiva sobre la desigualdad educativa en México}

México es un país ampliamente diverso tanto en lo natural como en lo económico y lo social. Estas diferencias han generado una multitud de escenarios educativos que hacen muy distintas las oportunidades de quienes habitan en el campo o la ciudad, en una entidad u otra, y de quienes viven en un estrato socioeconómico u otro.

Los avances acontecidos en materia educativa en México durante los últimos 50 años han modificado de manera profunda el perfil demográfico del mexicano. Sin embargo, los indicadores en materia de educación siguen siendo bajos cuando se realizan comparaciones internacionales entre México y otros países con un desarrollo económico similar. Aunado a esto, hay que agregar una persistente desigualdad regional y la gran heterogeneidad de los escenarios educativos y demográficos del país (Giorguli, Vargas, Salinas, Hubert \& Potter, 2010).

Diversos trabajos han generado evidencia empírica sobre la desigualdad educativa en México y la han caracterizado para diferentes momentos históricos y regiones. Los primeros trabajos clásicos sobre el tema se plantearon como objetivo inicial dimensionar a la desigualdad educativa y realizar comparativos regionales sobre su comportamiento; posteriormente, estos estudios pasarían de una visión preponderantemente cuantitativa a una más cualitativa, centrada en las características de los involucrados y en la forma en que los aprendizajes estaban distribuyéndose entre la población; es decir privilegiando la obtención de saberes y habilidades sobre la obtención de credenciales educativas.

Bracho (1995), en un estudio que abarcó de 1960 a 1990, encontró que si bien la incorporación al tejido escolar se incrementó de manera importante y disminuyó la cantidad de personas excluidas del sistema educativo, los problemas de rezago permanecen, particularmente si se contrastan los resultados alcanzados contra la norma constitucional. Asimismo señala que la gravedad de los problemas está altamente diferenciada, al realizar un comparativo entre las entidades federativas, encontrando 
una diferencia de casi tres años de escolaridad entre las entidades más avanzadas y las más rezagadas, demostrándose así una profunda desigualdad interna en el país, determinada en buena medida por la fuerza de sus sistemas educativos y por su capacidad para atraer a la población más educada. Señala también que toma alrededor de diez años el incrementar la escolaridad promedio de la población en un grado, lo que implicaría un esfuerzo de casi treinta años para que las entidades más rezagadas alcanzaran el nivel educativo de las más avanzadas.

Martínez (1992), utilizando datos que abarcaron de 1970 a 1990, encontró como las entidades más desiguales en materia educativa a Chiapas, Oaxaca y Guerrero, seguidos de Guanajuato, Zacatecas y Michoacán. En este mismo estudio, Martínez (1992) enfatiza la relación existente entre los indicadores educativos (tales como la escolaridad) y los indicadores de desarrollo socioeconómico de estas entidades. Años después, Martínez (2002) actualizaría este estudio para abarcar el periodo 1970-2000, encontrando que, medida a través del Coeficiente de Gini, la desigualdad educativa en México disminuyó de forma tal que la distancia relativa entre las entidades federativas permanece igual a través del tiempo. Por ello, es posible decir que estos avances son de carácter inercial, es decir, son el resultado del proceso general de crecimiento del país y no de políticas compensatorias establecidas.

Salgado y Rodríguez (2012), con datos para el periodo 1995-2005, encuentran los niveles de desigualdad más elevados en los estados de Chiapas, Puebla, Oaxaca y Guerrero, y destacan como las entidades más igualitarias a Quintana Roo y el Estado de México.

Navarro y Favila (2013), en su estudio para el periodo 1990-2010, encuentran las condiciones de desigualdad educativa más severas en los estados de Chiapas, Guerrero, Oaxaca y Michoacán. En este estudio confirman de manera empírica que, aunque todas las entidades se volvieron más igualitarias a través del tiempo, la distancia de desigualdad entre los estados permaneció prácticamente constante.

Estas condiciones de desigualdad entre entidades federativas se reproducen a escala municipal y ponen en evidencia la estratificación sociodemográfica prevaleciente. Ejemplos de ello pueden encontrarse en trabajos como el de Rodríguez (2009) quien, al analizar el caso del estado de Hidalgo, encuentra que las oportunidades de acceder a la educación benefician de manera diferente a los distintos grupos sociales por razones vinculadas al género, la edad y el origen étnico de los beneficiarios; los Programas de las Naciones Unidas para el Desarrollo (PNUD, 2013) encuentran brechas de desigualdad educativa considerables entre la población indígena y no-indí- 
gena de México, señalando que la población indígena obtuvo en 2010 solamente 39\% de la escolaridad que obtienen los habitantes no-indígenas. En otro trabajo, Tapia y Valenti (2016) expresan que la desigualdad percibida entre las entidades federativas es producto de la desigualdad intraescolar (la estratificación de la población escolar de acuerdo con sus características socioeconómicas) e interescolar (la que existe entre las diferentes escuelas según su modalidad o su ubicación), por lo que las entidades sólo podrán superar su condición de desigualdad actual en la medida que puedan resolver las diferencias que distinguen a unas escuelas de otras.

Estos trabajos muestran una serie de características comunes: señalan que existen en México entidades relativamente avanzadas y rezagadas separadas por brechas que sólo pueden abatirse en grandes periodos de tiempo. Entre las rezagadas es posible contar a Chiapas, Oaxaca, Guerrero y Michoacán; la escala estatal no permite un análisis completo de la problemática, ya que ésta se intensifica conforme el enfoque se mueve hacia segmentos más pequeños, tales como los municipios o los centros escolares; y la prevalencia de las condiciones socioeconómicas y de pertenencia a grupos vulnerables como determinantes de la desigualdad.

\subsection{La relación entre la desigualdad educativa y la desigualdad en la distribución del ingreso}

En la literatura del tema se ha relacionado a la desigualdad educativa con una variedad de efectos nocivos en la sociedad y en la economía. Una de las inquietudes académicas sobre estas consecuencias se relaciona con conocer si la desigualdad educativa juega un papel activo en la reproducción de otras formas de desigualdad, por ejemplo, la desigualdad en la distribución del ingreso.

Huerta (2012) comenta que los determinantes de la desigualdad en la distribución de los ingresos pueden alinearse bajo dos perspectivas teóricas básicas: la teoría del excedente, la cual indica que los ingresos dependen de la propiedad de los activos que intervienen en la producción, y la teoría de la productividad, la cual indica que los individuos más productivos son remunerados con mayores percepciones. Esto va de acuerdo con la Teoría del Capital Humano, en la cual una mayor preparación de la fuerza laboral genera una mayor productividad de la misma y, en consecuencia, ésta tendría que ser recompensada con mayores remuneraciones.

Pese a que la relación entre la educación de las personas y sus ingresos es ampliamente aceptada, existe un menor número de trabajos actualizados sobre la relación 
existente entre las desigualdades en la distribución de la educación y de los ingresos. Para el caso de México, algunos de los trabajos que han abordado dicha relación son los de Székely (1995) — donde el autor encuentra como determinante fundamental de la distribución del ingreso al número de años de educación de la población-o el de Bracho (1995), donde la autora aporta que las distribuciones de bienes como el ingreso y la educación se encuentran vinculadas y que no es posible acceder a cambios sustanciales en una de estas desigualdades dejando de lado a la otra.

Por otro lado, Martínez, Hernández y Valles (2014) revelan que, a pesar de que una mayor escolaridad se encuentra positivamente relacionada con mejores percepciones, esto no significa que la misma tenga un efecto significativo en la distribución del ingreso; de hecho, mientras la desigualdad educativa tiende a disminuir a través del tiempo, esto no sucede necesariamente con la desigualdad del ingreso, la cual puede subir o bajar.

Otros determinantes que podrían influir en este comportamiento son las capacidades de los trabajadores adquiridas por la capacitación o la experiencia, el sector de la actividad económica donde se desempeñan los individuos, la discriminación, la exclusión y la situación de los ciclos económicos (Vargas, 2006).

Con base en lo anterior, surge la inquietud de corroborar con datos recientes si la relación entre las variables planteadas existe y si la misma es significativa para el caso de las entidades federativas.

\section{Estado de la escolaridad en las entidades federativas mexicanas}

Como se comentó anteriormente, la educación en México está marcada por profundas brechas de desigualdad que generan una distinción clara entre las entidades avanzadas y las entidades rezagadas.

Si bien los resultados del Coeficiente de Gini proveen una medida más exacta de la magnitud de la desigualdad, una descripción de los datos que arroja la Encuesta Intercensal (Instituto Nacional de Estadística y Geografía [INEGI], 2015) también resulta reveladora.

La Tabla 1 muestra los años promedio de escolaridad de la población de 15 años y más por entidad federativa. Se observa que el promedio nacional de este indicador es de 9.16 años y que dieciocho de las 32 entidades consideradas se encuentran por encima de este promedio. 
Tabla 1. Años promedio de escolaridad por entidad federativa en 2015 para la población de 15 años y más

\begin{tabular}{cccccc}
\hline No. & Entidad & Promedio & No. & Entidad & Promedio \\
\hline 1 & Distrito Federal & 11.12 & 17 & Tlaxcala & 9.27 \\
\hline 2 & Nuevo León & 10.27 & 18 & Jalisco & 9.25 \\
\hline 3 & Sonora & 10.00 & 19 & Nayarit & 9.16 \\
\hline 4 & Baja California Sur & 9.91 & 20 & Durango & 9.15 \\
\hline 5 & Coahuila & 9.90 & 21 & Campeche & 9.14 \\
\hline 6 & Baja California & 9.76 & 22 & Yucatán & 8.82 \\
\hline 7 & Aguascalientes & 9.73 & 23 & San Luis Potosí & 8.82 \\
\hline 8 & Quintana Roo & 9.62 & 24 & Hidalgo & 8.72 \\
\hline 9 & Sinaloa & 9.58 & 25 & Zacatecas & 8.63 \\
\hline 10 & Querétaro & 9.55 & 26 & Puebla & 8.49 \\
\hline 11 & Colima & 9.53 & 27 & Guanajuato & 8.38 \\
\hline 12 & México & 9.53 & 28 & Veracruz & 8.20 \\
\hline 13 & Tamaulipas & 9.51 & 29 & Michoacán & 7.93 \\
\hline 14 & Chihuahua & 9.47 & 30 & Guerrero & 7.80 \\
\hline 15 & Tabasco & 9.31 & 31 & Oaxaca & 7.52 \\
\hline 16 & Morelos & 9.30 & 32 & Chiapas & 7.29 \\
\hline \multicolumn{7}{c}{ Estados Unidos } & 9.16 & & & \\
\hline
\end{tabular}

Fuente: Elaboración propia con datos de la Encuesta Intercensal (INEGI, 2015).

La entidad mejor posicionada en 2015 fue el Distrito Federal (D.F.) y le siguieron en importancia Nuevo León y Sonora. El líder nacional (D.F.) se encuentra por encima de la media nacional por casi dos años de escolaridad. Esto implica que en el D.F. la escolaridad promedio de la población de 15 años y más es comparable con llegar al último grado del bachillerato y no concluirlo. Por otro lado, la entidad con el menor desempeño en este indicador fue Chiapas con un valor de 7.29 años. Esto significa una escolaridad promedio equivalente a alcanzar el segundo grado de secundaria y no terminarlo. En consecuencia, la distancia en escolaridad entre el líder nacional y la entidad más rezagada es de casi 3.8 años, una diferencia considerable si se toma en 
cuenta que ambas entidades se encuentran sometidas a ambientes de política pública muy similares.

Otras entidades que obtuvieron valores bajos en este indicador fueron Oaxaca, Guerrero, Michoacán y Veracruz, que consiguieron valores entre 7.5 y 8.2 años. Estas cifras brindan una primera aproximación sobre las diferencias educativas existentes entre entidades.

Otro aspecto relevante para aproximarse a este escenario es la cantidad de personas que quedan excluidas de obtener escolaridad. En este sentido, la Figura 1 muestra los porcentajes de la población que declaró no tener escolaridad alguna en la Encuesta Intercensal (INEGI, 2015); en ella se muestran sólo las entidades que resultaron mejor y peor posicionadas.

Como se aprecia, existieron coincidencias importantes con lo encontrado en la Figura 1. Las entidades con la mayor proporción de su población (de 15 años y más) sin escolaridad fueron Chiapas, Guerrero, Oaxaca, Veracruz, Michoacán y Puebla. Chiapas fue nuevamente el caso de mayor rezago con un $14.55 \%$, es decir un segmento bastante representativo de su población.



Figura 1. Proporción de la población de 15 años y más sin escolaridad por Entidad Federativa

Fuente: Elaboración propia con datos de la Encuesta Intercensal del INEGI (2015). 
Situaciones similares se registraron en Guerrero, con $13.25 \%$, y Oaxaca, con $11.84 \%$. Una situación muy distinta sucedió en entidades como el Distrito Federal, Nuevo León, Coahuila, Sonora y Baja California, cuya población en este indicador se encontró entre el 2 y el $3 \%$, lo que implica que en ellas prácticamente el total de la población tiene acceso a alguna escolaridad. La brecha de desigualdad en este indicador, entre las entidades avanzadas y las rezagadas es de casi 7 veces, situación que marca una severa brecha entre ellas.

\section{El Coeficiente de Gini para la educación}

Un índice de desigualdad resume la manera como se distribuye una variable entre un conjunto de individuos. Una de las medidas utilizadas con más frecuencia para ello es el Coeficiente de Gini, el cual se construye al comparar la distribución empírica que se forma con los datos observados y la línea de igualdad perfecta que supone la distribución teórica (Medina, 2001).

Al ser la educación un concepto difícil de definir y, por lo tanto, de medir, una forma de aproximarse a la medición de su desigualdad es representándola a través de la escolaridad.

Thomas, Wang y Fan (2001) proponen la siguiente fórmula para adaptar el coeficiente de Gini para ser utilizado en el estudio de la desigualdad en la educación:

$$
E_{L}=\left(\frac{1}{\mu}\right) \sum_{i=2}^{n} \sum_{j=1}^{i-1} p_{i}\left|y_{i}-y_{j}\right| p_{j}
$$

Donde:

$E_{L}$ : Representa el coeficiente de Gini para la educación, es decir el grado de desigualdad existente entre los miembros de la población.

$\mu$ : Es el promedio de años de escolaridad para la población estudiada.

$P_{i}$ y $P_{j}$ : Representan las proporciones de la población que posee un nivel de educación determinado $(n)$.

$Y_{i}$ y $Y_{j}:$ Representan el número de años de escolaridad requeridos para alcanzar un nivel de educación $(n)$.

$n$ : Es el número de divisiones que se proponen para segmentar los diversos niveles de escolaridad en una sociedad. Para este caso la segmentación se realizó en 
los siguientes niveles: Sin Escolaridad, Preescolar, Primaria Completa, Secundaria Incompleta, Secundaria Completa, Media Superior y Superior.

El coeficiente de Gini puede alcanzar valores entre o y 1, donde o representa la perfecta igualdad y 1 representa la perfecta desigualdad. En consecuencia, es deseable para una sociedad el obtener valores bajos de este indicador y que los mismos disminuyan a través del tiempo; esto significaría el logro de avances en el fortalecimiento del sistema educativo y en las condiciones del entorno que permitan a la población acceder a una mayor educación.

\section{Resultados de los instrumentos de medición}

Se midieron los coeficientes de Gini para la educación de las entidades federativas de la República Mexicana utilizando para ello los datos de la Encuesta Intercensal del INEGI (2015). Esta encuesta tiene por objetivo el actualizar el estado de la información sociodemográfica de México entre los censos de 2010 y el que se realizará en 2020. La Encuesta Intercensal abarcó una muestra de 6.1 millones de viviendas distribuidas por todo el país y es representativa a nivel nacional, estatal, municipal y de localidades con 50,000 habitantes o más (INEGI, 2015).

La medición se realizó para la población total de 25 años y más. Los resultados obtenidos se plasman en la Tabla 2 ordenados del valor más bajo al más alto de acuerdo con la columna "población general".

Los resultados sugieren que la entidad en la que la educación fue más igualitaria en 2015 fue el Distrito Federal, una entidad altamente urbana, con la menor proporción de población sin escolaridad y con la mayor escolaridad promedio del país. Le siguieron los estados de Nuevo León, Sonora, Coahuila y Baja California. En el fondo de esta clasificación se encontró el estado de Chiapas. Otros valores relativamente altos se ubicaron en Guerrero, Oaxaca, Veracruz y Michoacán. Es notable que Chiapas obtuviera un coeficiente de casi el doble al obtenido por el Distrito Federal, situación que pone de manifiesto las importantes diferencias educativas que existen entre las entidades del país y el profundo rezago relativo en el que se encuentra la entidad. 
Tabla 2. Coeficientes de Gini para la educación en las entidades federativas mexicanas en 2015 para la población de 25 años y más

\begin{tabular}{cccccc}
\hline No. & Entidad & $\begin{array}{c}\text { Población } \\
\text { general }\end{array}$ & No. & Entidad & $\begin{array}{c}\text { Población } \\
\text { general }\end{array}$ \\
\hline 1 & Distrito Federal & 0.179 & 17 & Tabasco & 0.221 \\
\hline 2 & Nuevo León & 0.186 & 18 & Sinaloa & 0.225 \\
\hline 3 & Sonora & 0.193 & 19 & Morelos & 0.227 \\
\hline 4 & Coahuila & 0.194 & 20 & Nayarit & 0.228 \\
\hline 5 & Baja California & 0.195 & 21 & Querétaro & 0.232 \\
\hline 6 & Baja California Sur & 0.200 & 22 & Hidalgo & 0.237 \\
\hline 7 & Durango & 0.202 & 23 & San Luis Potosí & 0.239 \\
\hline 8 & Aguascalientes & 0.202 & 24 & Yucatán & 0.244 \\
\hline 9 & Estado de México & 0.205 & 25 & Guanajuato & 0.250 \\
\hline 10 & Chihuahua & 0.205 & 26 & Campeche & 0.251 \\
\hline 11 & Tamaulipas & 0.207 & 27 & Puebla & 0.260 \\
\hline 12 & Tlaxcala & 0.208 & 28 & Michoacán & 0.268 \\
\hline 13 & Quintana Roo & 0.210 & 29 & Veracruz & 0.269 \\
\hline 14 & Zacatecas & 0.217 & 30 & Oaxaca & 0.297 \\
\hline 15 & Jalisco & 0.218 & 31 & Guerrero & 0.318 \\
\hline 16 & Colima & 0.221 & 32 & Chiapas & 0.327 \\
\hline
\end{tabular}

Fuente: Cálculos propios con base en datos de INEGI, 2015.

Con la información de la Tabla 3, se generaron cuartiles que permitieran elaborar un mapa para ubicar geográficamente las zonas en las que la desigualdad se manifestó de manera más intensa. Para la elaboración de los cuartiles se calculó la diferencia entre la entidad mejor evaluada y la peor evaluada, y después se dividió entre cuatro para crear un igual número de rangos.

En la Figura 2 muestra los resultados obtenidos. En color blanco aparecen las entidades que obtuvieron coeficientes de Gini entre 0.179 y 0.216 ; en color gris claro aparecen las entidades evaluadas con valores entre 0.216 y 0.253 ; en color gris se señalan los estados con coeficientes entre 0.253 y 0.29 , y en color negro los que obtuvieron cifras superiores a 0.29 . 


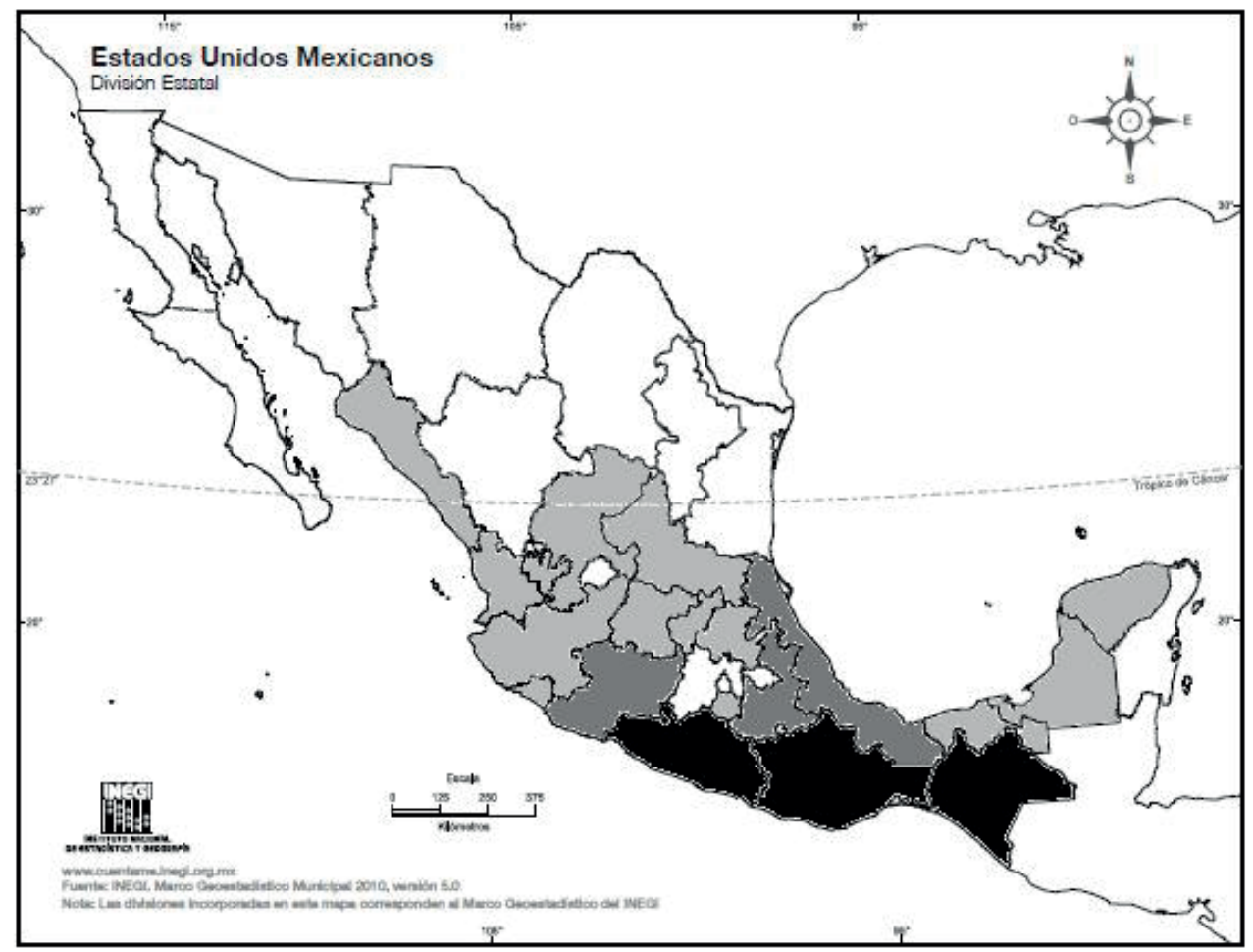

Coeficiente entre 0.179 y 0.216

Coeficiente entre 0.216 y 0.253

Coeficiente entre 0.253 y 0.29

Coeficiente superior a 0.29

Figura 2. Coeficientes de Gini para la educación en los estados mexicanos para la población de ambos géneros de 25 años y más en 2015

Fuente: Elaboración propia con base en la Tabla 3.

Como puede observarse en la Figura 2, la desigualdad educativa en México presenta ciertos patrones geográficos en el territorio nacional. Si bien el realizar los cálculos a escala estatal hace que no pueda distinguirse la diversidad que cada estado encierra en su interior, sí es posible distinguir ciertas pautas territoriales, encontrando las condiciones más igualitarias en los estados fronterizos del norte de la República y en la capital del país y su zona conurbada. Una situación distinta se ubica conforme se 
observa el mapa hacia el sur, particularmente en los estados colindantes con el Océano Pacífico, en los cuales se registraron las condiciones de desigualdad educativa más acentuadas del país.

Existe un conjunto de características comunes en los estados más rezagados que dan indicios sobre las condiciones que propician este comportamiento. Guerrero, Oaxaca y Chiapas comparten los tres últimos lugares en empleo formal (INEGI, 2016), en el PIB per cápita no minero (Instituto Nacional para el Federalismo y el Desarrollo Municipal [INAFED], 2013), en Ingreso Bruto per Cápita (INAFED, 2013) y en ingreso promedio trimestral de los hogares (INEGI, 2014). Ocupan también los tres últimos lugares en indicadores tales como el Índice de Desarrollo Humano del PNUd (INAFED, 2013) y el Índice de Marginación (Consejo Nacional de Población [CONAPO], 2015) y son además los tres estados con la mayor proporción de su población en situación de pobreza (Consejo Nacional de Evaluación de la Política de Desarrollo Social [CONEVAL], 2014) y con la mayor proporción de su población habitando en zonas rurales (en Guerrero, casi 50\%, en Chiapas, 57\%, en Oaxaca, 61\%) (conevaL, 2014) lo cual dificulta que la infraestructura educativa existente y los medios de comunicación para llegar a ella sean debidamente aprovechados por los demandantes de servicios educativos y que el acceso a estas oportunidades tenga un costo considerablemente mayor para los hogares rurales.

Lo anterior lleva a pensar que, en un ambiente donde las políticas educativas son similares de una entidad a otra, son las carencias socioeconómicas las que perpetúan la distancia de desigualdad educativa entre los estados.

\subsection{La distribución de los ingresos en los hogares mexicanos por entidad federativa y su relación con la distribución de la escolaridad}

Después de observar el estado que guarda el Coeficiente de Gini para la educación en los estados mexicanos, se procedió a probar si este indicador se encuentra relacionado con la distribución del ingreso de los mismos.

Para lo anterior se utilizaron datos provenientes de la Encuesta Nacional de Ingresos y Gastos de los Hogares de 2014 publicada por el INEGI. La Figura 3 muestra un resumen de los datos encontrados: 


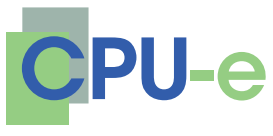

Desigualdad educativa y su relación con la distribución

del ingreso en los estados mexicanos

Antonio Favila Tello, José César Lenin Navarro Chávez

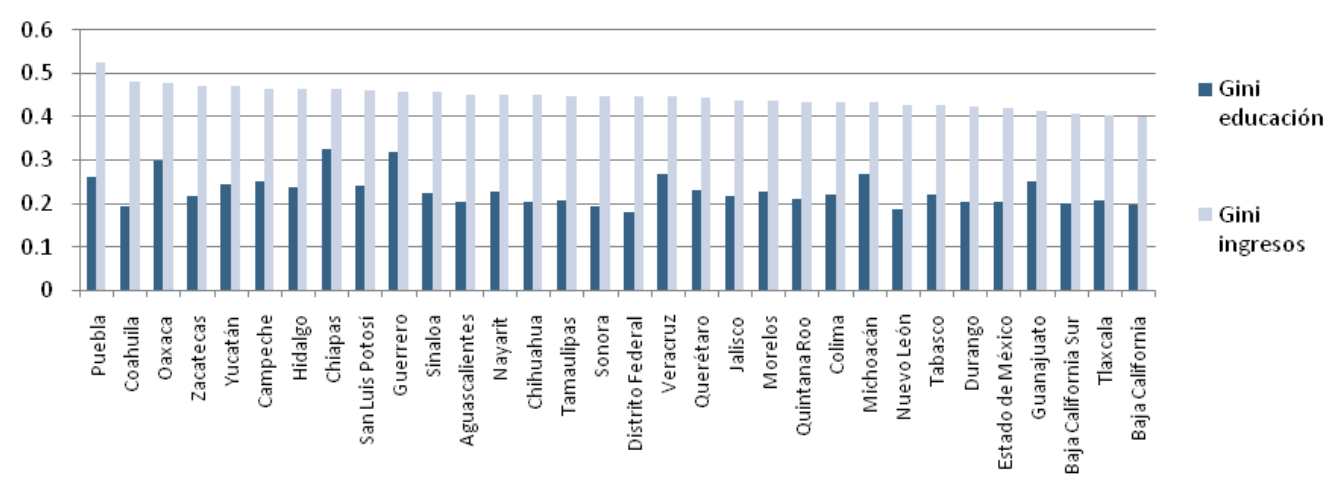

\section{Figura 3. Comparativo entre el coeficiente de Gini para la educación y para el ingreso}

Fuente: Elaboración propia con base en datos de INEGI, 2014, y de la Tabla 2.

En materia de ingreso, el estado con la distribución más desigual fue Puebla, con un Coeficiente de Gini de 0.52; le siguieron Coahuila, Oaxaca y Zacatecas. En Puebla, por ejemplo, el ingreso promedio trimestral de los hogares más ricos es casi 35 veces más alto que el de los hogares más pobres; en Coahuila esta cifra es de 27 veces, en Oaxaca de 26 y en Zacatecas de 24. Por otro lado, los estados donde el ingreso de los hogares se distribuyó de manera más igualitaria fueron Baja California, Tlaxcala y Baja California Sur.

Como muestra la Figura 3, lo encontrado mediante las gráficas no coincide de manera exacta con lo esperado, es decir, no existe una correspondencia perfecta entre los estados con el mayor coeficiente de Gini para la Educación y para el Ingreso, salvo por el caso de Oaxaca que en ambos indicadores se encontró en los últimos lugares. Entidades como el Distrito Federal, el más igualitario en educación, ocupa el lugar 16 en cuanto a distribución del ingreso; por otro lado, Chiapas, el más desigual en educación, resultó en el lugar 25.

Guerrero y Chiapas, pese a tener los ingresos trimestrales promedio por hogar más bajos del país, no aparecen entre los más desiguales, esto debido a que sus ingresos son bajos para todos los deciles, es decir, los hogares de todos los estratos tienen ingresos relativamente bajos cuando se les compara con los hogares del resto del país. Por ejemplo, un hogar que está en el decil más alto en Chiapas tiene ingresos promedio de casi un tercio de lo que obtiene un hogar del decil más alto en el Distrito Federal o Nuevo León. 
Si bien el coeficiente de Gini puede dar una idea sobre la desigualdad en la distribución del ingreso dentro del territorio, una mayor igualdad no refleja necesariamente mejores condiciones, simplemente refleja que los sujetos son más similares entre sí, sea para bien o para mal.

Para probar la hipótesis sobre si una mayor desigualdad educativa es determinante de una mayor desigualdad en la distribución del ingreso, se procedió a realizar una regresión lineal por mínimos cuadrados ordinarios entre estas dos variables. Los resultados obtenidos se muestran en la Tabla 3.

\section{Tabla 3. Resultados de la regresión entre el Coeficiente de Gini para los ingresos (2014) el Coeficiente de Gini para la educación (2015)}

Dependent Variable: GINI_INGRESOS

Method: Least Squares

Date: 09/05/16 Time: 16:13

Sample: 132

Included observations: 32

\begin{tabular}{lclll}
\hline \multicolumn{1}{c}{ Variable } & Coefficient & Std. Error & t-Statistic & Prob. \\
\hline \multicolumn{1}{c}{ GINI_EDUCACION } & $\mathbf{0 . 2 8 7 5 6 3}$ & $\mathbf{0 . 1 1 9 6 1 1}$ & $\mathbf{2 . 4 0 4 1 5 6}$ & $\mathbf{0 . 0 2 2 6}$ \\
\multicolumn{1}{c}{$\mathbf{0}$} & $\mathbf{0 . 3 8 0 3 3 0}$ & $\mathbf{0 . 0 2 7 7 4 7}$ & $\mathbf{1 3 . 7 0 7 0 4}$ & $\mathbf{0 . 0 0 0 0}$ \\
\hline R-squared & 0.161542 & Mean dependent var & 0.446245 \\
\hline Adjusted R-squared & 0.133593 & S.D. dependent var & 0.025934 \\
\hline S.E. of regression & 0.024139 & Akaike info criterion & -4.549492 \\
\hline Sum squared resid & 0.017481 & Schwarz criterion & -4.457884 \\
\hline Log likelihood & 74.79188 & Hannan-Quinn criter. & -4.519127 \\
\hline F-statistic & 5.779966 & Durbin-Watson stat & 0.390635 \\
\hline Prob(F-statistic) & 0.022591 & & \\
\hline
\end{tabular}

Fuente: Cálculos propios con datos de la Tabla 2 y la de la Encuesta Nacional de Ingresos y Gastos de los Hogares del INEGI, 2014.

Los resultados muestran que las variables se encuentran relacionadas de manera positiva, es decir, a una mayor desigualdad educativa le corresponde una mayor desigualdad en la distribución del ingreso; sin embargo, su influencia es relativamente menor 
dados los bajos valores del $\mathrm{R}^{2} \mathrm{y}$ del coeficiente asociado a la variable independiente. Esto sugiere que, al tratarse de un modelo con una sola variable independiente, el resto de comportamiento de la distribución del ingreso de los hogares se debe a otros determinantes (ver Anexos 1 y 2).

\section{Conclusiones}

A lo largo del presente trabajo se describieron los resultados obtenidos para el Coeficiente de Gini para la educación en las entidades federativas de México con datos para 2015. Los resultados obtenidos confirman lo encontrado en trabajos anteriores sobre el tema: la existencia de profundas brechas de desigualdad entre entidades y el rezago de estados como Chiapas, Oaxaca y Guerrero que han estado al fondo de esta clasificación desde la segunda mitad del siglo xx.

Se confirma también que la desigualdad educativa, medida a partir de la distribución de la escolaridad, tiende a disminuir a través del tiempo y que la distancia de desigualdad relativa entre entidades tiende a permanecer constante pese a las políticas instrumentadas para incrementar la escolaridad de la población y pese al crecimiento del Sistema Educativo Nacional.

Se logró demostrar la hipótesis que indica que la distribución del ingreso en las entidades federativas se encuentra determinada por su desigualdad educativa. No obstante, a pesar de que el modelo por mínimos cuadrados ordinarios cumple con los supuestos para ser válido, el coeficiente asociado a la variable es significativo y presenta el signo esperado, los datos sugieren que el poder explicativo de la variable independiente es limitado. Una de las razones de ello es que el rango en el que fluctúan los coeficientes de Gini para la educación es más amplio que el rango en el que fluctúa el Gini del ingreso. La distancia entre el Gini educativo de la entidad más avanzada y la más atrasada es de casi $88 \%$, mientras que la distancia de desigualdad en el Gini del ingreso entre la entidad más avanzada y la más atrasada es de alrededor de $32 \%$. Esto sugiere que los escenarios de distribución del ingreso entre las entidades federativas son más similares entre sí que los altamente contrastantes escenarios educativos.

Entidades que son ejemplares en materia de igualdad educativa, como el Distrito Federal, Nuevo León o Sonora, ciertamente siguen siendo bastante desiguales en materia de ingreso; por otro lado, entidades como Chiapas u Oaxaca, las más desiguales en materia educativa, poseen desigualdades en el ingreso similares a las de estados 
como Campeche, San Luis Potosí o Hidalgo, las cuales poseen, en general, indicadores educativos más favorables. Lo anterior sugiere que los beneficios de los avances educativos están teniendo dificultades para trasladarse a otros ámbitos de la vida de la sociedad, como el mercado laboral o las retribuciones salariales.

Otra causa de estos comportamientos puede relacionarse con las dificultades inherentes a la medición de la distribución del ingreso a través de encuestas, ya que puede suceder que las familias tiendan a mentir sobre sus ingresos reales y que la información de los hogares más ricos sea casi imposible de conseguir (para una discusión más abundante sobre esto véase Esquivel, 2015).

Futuras líneas de investigación podrían abundar sobre la relación de estos tipos de desigualdad agregando otras variables de control e indagando con periodos de tiempo más largos para fortalecer el conocimiento que existe sobre esta relación. Algunas posibles causas podrían relacionarse con la estructura económica y/o demográfica de cada entidad, con el grado de informalidad del empleo en cada estado, con el aprovechamiento de la educación (y no solamente con los avances de la escolaridad) y con el uso de métodos alternativos de medición del ingreso de los hogares (por ejemplo, con datos provenientes del Sistema de Cuentas Nacionales).

\section{Lista de referencias}

Acemoglu, D., \& Autor, D. (s.f.). Lectures in labor economics. Cambridge: MIT Press. Bracho, T. (1995). Distribución y desigualdad educativa en México. Revista Estudios Sociológicos, 8(37), 25-37.

Consejo Nacional de Evaluación de la Política de Desarrollo Social. (2014). Medición de la pobreza, Estados Unidos Mexicanos, 2014. Evolución de la pobreza y pobre$z a$ extrema nacional y en entidades federativas, 2010, 2012 y 2014. Recuperado de http://www.coneval.org.mx/Medicion/Paginas/PobrezaInicio.aspx

Consejo Nacional de Población. (2015). Datos abiertos del Índice de Marginación. Recuperado de http://www.conapo.gob.mx/es/CONAPo/Datos_Abiertos del Indice de Marginacion

Eicher, J. (1988). Treinta años de economía de la educación. Ekonomiaz, Revista Vasca de Economía, 12, 11-38

Esquivel, G. (2015). Desigualdad extrema en México. México: Oxfam.

Giorguli, S., Vargas, E., Salinas, V., Hubert, C., \& Potter, J. (enero-abril, 2010). La di- 
námica demográfica y la desigualdad educativa en México. Estudios Demográficos y Urbanos, 25(1), 7-44.

Huerta, R. (2012). Pobreza, distribución del ingreso y renta básica. Economía UNAM, 9(26), 68-81.

Instituto Nacional de Estadística y Geografía. (2014). Encuesta Nacional de Ingresos y Gastos de los Hogares. Recuperado de http://www.inegi.org.mx/est/contenidos/Proyectos/encuestas/hogares/regulares/enigh/

Instituto Nacional de Estadística y Geografía. (2015). Encuesta Intercensal 2015. Recuperado de http://www.inegi.org.mx/est/contenidos/Proyectos/encuestas/ hogares/especiales/ei2015/

Instituto Nacional de Estadística y Geografía. (2016). Tasas de informalidad laboral y ocupación en el sector informal por entidad federativa y sexo. Encuesta Nacional de Ocupación y Empleo. Recuperado de http://www.beta.inegi.org.mx/contenidos/proyectos/enchogares/regulares/enoe/tabulados/15ymas/informalidad tasas $15 y$ mas xls.zip

Instituto Nacional para el Federalismo y el Desarrollo Municipal. (2013). Indicadores Municipales. Socioeconómico Municipal. Recuperado de http://www.inafed. gob.mx/es/inafed/Socioeconomico_Municipal

Instituto Nacional para la Evaluación de la Educación. (2014). El derecho a una educación de calidad. Informe 2014. México: Autor. Recuperado de http://publicaciones.inee.edu.mx/buscadorPub/P1/D/239/P1D239.pdf

Latapí, P. (1983). La desigualdad educativa en México. En C. Bazdresch \& J. Carabaña (Coords.) Desigualdad y equidad en España y México (pp. 199-224). México: Instituto de Cooperación Iberoamericana/El Colegio de México.

Martínez, F. (1992). La desigualdad educativa en México. Revista Latinoamericana de Estudios Educativos, 22(2), 59-120.

Martínez, F. (2002). Nueva visita al país de la desigualdad. La distribución de la escolaridad en México, 1970-2000. Revista Mexicana de Investigación Educativa, 7(16), 415-443.

Martínez, J., Hernández, J., \& Valles, H. (2014). Educación y desigualdad del ingreso en municipios del estado de Chihuahua. Revista de Investigación Educativa de la Rediech, 9, 77-83. Recuperado de http://www.rediech.org/inicio/index.php/biblioteca/articulos/item/download/37_82f4fba661c9757704636b 7b88944059

Medina, F. (2001). Consideraciones sobre el índice de Gini para medir la concentración del 
ingreso (Serie 9. Estudios estadísticos y prospectivos). Santiago de Chile: Comisión Económica para América Latina y el Caribe.

Meschi, E., \& Scervini, F. (2010). A new dataset of educational inequality. Empirical Economics, 47 (2), 695-716. doi:10.1007/soo181-013-0758-6

Muñoz, C. (2003). Origen y consecuencias de las desigualdades educativas. Investigaciones realizadas en América Latina sobre el problema. México: Fondo de Cultura Económica.

Navarro, J. C., \& Favila, A. (2013). La desigualdad de la educación en México, 19902010: el caso de las entidades federativas. Revista Electrónica de Investigación Educativa, 15(2), 21-33. Recuperado de http://redie.uabc.mx/redie/article/ view/441/605

Ordaz, J. (2007). México: capital humano e ingresos. Retornos a la educación, 1994-2005. México: Comisión Económica para América Latina y el Caribe-Organización de las Naciones Unidas.

Programas de las Naciones Unidas para el Desarrollo. (2013). Índice de equidad educativa indígena. Informe de resultados para México, sus Estados y Municipios, 2010. México: Autor.

Rodríguez, C. (septiembre de 2009). Diferencias en las oportunidades educativas de las personas según su origen social en el estado de Hidalgo. En Consejo Mexicano de Investigación Educativa (Ed.), Memoria electrónica del X Congreso $\mathrm{Na}$ cional de Investigación Educativa [CD-ROM]. México: Editor. pp. 1-14.

Salgado, J., \& Rodríguez, K. (enero-junio, 2012). La desigualdad en educación en México por entidad federativa 1995-2005. Revista Educación, 36(1), 1-32.

Subirats, J., Gomá, R., \& Brugué, J. (2005). Análisis de los factores de exclusión social. Bilbao: Fundación BBVA.

Székely, M. (1995). Aspectos de la desigualdad en México. El trimestre económico, $62(246), 201-243$.

Tapia, L., \& Valenti, G. (2016). Desigualdad educativa y desigualdad social en México. Nuevas evidencias desde las primarias generales en los estados. Perfiles educativos, $38(151), 32-54$.

Thomas, V., Wang, Y., \& Fan, X. (2001). Measuring education inequality: Gini Coefficients of Education. Washington: World Bank Institute.

Vargas, G. (2006). Introducción a la teoría económica. México: Pearson Educación. 


\section{Anexos}

\section{Anexo 1. Prueba de normalidad}

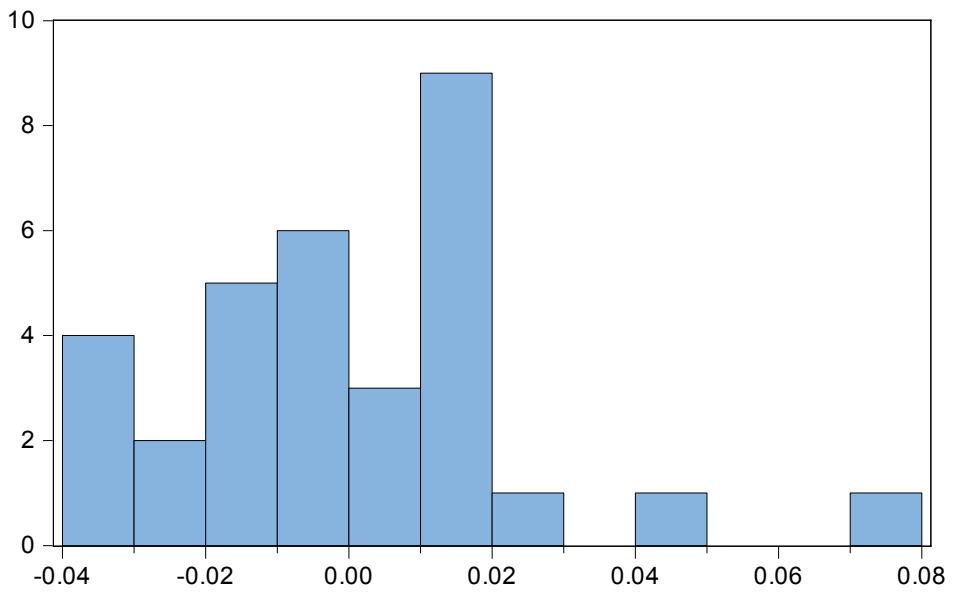

Series: Residuals

Sample 132

Observations 32

Mean

$9.58 \mathrm{e}-17$

Median

$-0.004168$

Maximum

0.071129

Minimum $\quad-0.038058$

Std. Dev. $\quad 0.023747$

Skewness $\quad 0.690863$

Kurtosis

4.082143

Jarque-Bera $\quad 4.106933$

Probability

0.128289 


\section{Anexo 2. Prueba de heterocedasticidad}

\begin{tabular}{|c|c|c|c|}
\hline \multicolumn{4}{|c|}{ Heteroskedasticity Test: White } \\
\hline F-statistic & 0.005536 & Prob. $F(1,30)$ & 0.9412 \\
\hline Obs*R-squared & 0.005904 & Prob. Chi-Square(1) & 0.9388 \\
\hline Scaled explained SS & 0.007996 & Prob. Chi-Square(1) & 0.9287 \\
\hline \multicolumn{4}{|l|}{ Test Equation: } \\
\hline \multicolumn{4}{|c|}{ Dependent Variable: $\operatorname{RESID} \wedge_{2}$} \\
\hline \multicolumn{4}{|l|}{ Method: Least Squares } \\
\hline \multicolumn{4}{|l|}{ Date: 09/05/16 Time: 16:36 } \\
\hline \multicolumn{4}{|l|}{ Sample: 132} \\
\hline \multicolumn{4}{|l|}{ Included observations: 32} \\
\hline Variable & Coefficient & t-Statistic & Prob. \\
\hline C & 0.000507 & 0.912964 & 0.3685 \\
\hline GINI_EDUCACION^² & 0.000729 & 0.074403 & 0.9412 \\
\hline R-squared & 0.000184 & Mean dependent var & 0.000546 \\
\hline Adjusted R-squared & -0.033143 & S.D. dependent var & 0.000974 \\
\hline S.E. of regression & 0.000990 & Akaike info criterion & -10.93642 \\
\hline Sum squared resid & $2.94 \mathrm{E}-05$ & Schwarz criterion & -10.84481 \\
\hline Log likelihood & 176.9827 & Hannan-Quinn criter. & -10.90606 \\
\hline F-statistic & 0.005536 & Durbin-Watson stat & 0.518749 \\
\hline Prob(F-statistic) & 0.941184 & & \\
\hline
\end{tabular}

